\title{
On the cumulant expansion peculiarity for partition function functional of the cluster de Gennes model
}

\author{
N.A.Korynevskii \\ Institute for Condensed Matter Physics \\ of the National Academy of Sciences of Ukraine, \\ 1 Svientsitskii Str., 79011 Lviv, Ukraine
}

Received February 16, 2000

The problem of the functional representation for cluster de Gennes model partition function within the collective variables method is discussed. Contrary to the usual Ising model case the coefficients of the obtained partition function functional (cluster cumulants) depend on temperature $T$ and transverse field $\Gamma$. Therefore there exists a rigorous limitation on the value of $\Gamma$ parameter at low temperatures. The equation for maximum value $\Gamma_{l}$, temperature and short-range intracluster interaction $V$ is obtained. The solutions of this equation have been found.

Key words: cluster de Gennes model, partition function functional, phase transitions

PACS: $05.60 .+W, 05.70 . L n, 05.20 . D d, 52.25 . D g, 52.25 . F i$

\section{Introduction}

The rapid progress in the second order phase transition theory which has taken place in the last third of the twentieth century was initiated by a revolutionary application of quantum field theory methods into this problem. The traditional statistic physics methods of investigation were, in some sense, relegated to the background. The idea has arised regarding the limited possibilities of statistical description of subtle peculiarities of the phase phenomenon. I.Yukhnovskii was the first who put modern second order phase transition theory on the rigorous base of statistical physics. There are two most important points in this theory. The first one: a statement about the basic distribution of variables connected with the order parameter in the phase transition point vicinity. And the second one: a method of layer by layer integration in the partition function functional which makes it possible to obtain in an explicit form all universal and non-universal characteristics of the system investigated [1]. 
The starting point of the theory is the expression for a partition function functional. For a usual Ising model which is characterized by one kind of interparticle interaction such a functional may be represented as an unlimited order exponential form with the constant coefficients. These coefficients are average values of the usual cumulants calculated with respect to the distribution of the external field Hamiltonian $h S^{z}\left(\vec{R}_{i}\right)$, the so-called "reference system". When a reference system is not so simple, for example for cluster ferroelectrics it contains short-range intracluster interactions and a transverse field, the partition function functional coefficients depend on these parameters, temperature and Matsubara's frequencies [2]. Naturally, a problem of their behaviour, namely a possibility to change its own sign, arrises. It must be noted that the sign of a higher order coefficient in the exponential form for a partition function functional determines the convergency of all functional integrals, that is the finity of the theory in a wide range of temperature and energy parameters. It seems very probable that the so-called anti-Curie points which occur in cluster ferroelectrics at low temperatures [3], arise due to the sign change in the higher order coefficient (fourth order) of partition function functional exponential form.

Therefore, the problem of correct calculation of cumulants of the cluster de Gennes model and their analysis from the point of convergency of total partition function functional is actual. For the systems described by long-range and shortrange potential and by the transverse field this task is not trivial. The aim of the present paper is to construct a total partition function functional for the cluster de Gennes model and to establish the conditions of its finity. The specific calculations will be done for a cluster system containing two particles in each cell $\left(f_{0}=2\right)$.

\section{Two-particle cluster system cumulants}

Let's consider the cluster de Gennes model Hamiltonian $[4,5]$

$$
\begin{aligned}
H= & \Gamma \sum_{q, f} S_{f}^{x}\left(\vec{R}_{g}\right)+h \sum_{q, f} S_{f}^{z}\left(\vec{R}_{q}\right)+V \sum_{q, f, f^{\prime}} S_{f}^{z}\left(\vec{R}_{q}\right) S_{f^{\prime}}^{z}\left(\vec{R}_{q}\right) \\
& +\sum_{q, q^{\prime}, f, f^{\prime}} J_{f f^{\prime}}\left(\vec{R}_{q}, \vec{R}_{q^{\prime}}\right) S_{f}^{z}\left(\vec{R}_{q}\right) S_{f^{\prime}}^{z}\left(\vec{R}_{q^{\prime}}\right) .
\end{aligned}
$$

Here $\Gamma$ and $h$ are transverse and longitudional external fields, respectively; $V$ is a pair intracluster interaction of particles; $J_{f f^{\prime}}\left(\vec{R}_{q}, \vec{R}_{q^{\prime}}\right)$ is a pair long-range potential; $S^{\alpha}$ are Pauli matricies; $1 \leqslant q \leqslant N$ is a cell-cluster number; $1 \leqslant f \leqslant f_{0}$ is the number of a particle in the cluster.

In the generalized transition operators representation

$$
Y_{\lambda}\left(\vec{R}_{q}\right)=\sum_{m} U_{\lambda m} X^{m}\left(\vec{R}_{q}\right)
$$

where $X^{m}\left(\vec{R}_{q}\right)$ are Hubbard-Stasyuk operators, $U_{\lambda m}$ are eigenfunctions of the total 
interaction matrix, (1.1) takes such a convenient form:

$$
H=\sum_{\lambda=1}^{2^{2 f_{0}}}\left\{\Lambda_{\lambda} \sum_{q} Y_{\lambda}\left(\vec{R}_{q}\right)-\frac{1}{2} \sum_{q, q^{\prime}} \Phi_{\lambda}\left(\vec{R}_{q}, \vec{R}_{q^{\prime}}\right) Y_{\lambda}\left(\vec{R}_{q}\right) Y_{\lambda}\left(\vec{R}_{q^{\prime}}\right)\right\}
$$

Here $\Lambda_{\lambda}$ are energy parameters of an isolated cluster (reference system), $\Phi_{\lambda}\left(\vec{R}_{q}, \vec{R}_{q^{\prime}}\right)$ are eigenvalues of long-range interaction matrix. For a two-particle $\left(f_{0}=2\right)$ interacting cluster system, for example, there are:

$$
\begin{array}{ll}
\Lambda_{1}=-2 h, & \Phi_{1}\left(\vec{R}_{q}, \vec{R}_{q^{\prime}}\right)=J_{11}\left(\vec{R}_{q}, \vec{R}_{q^{\prime}}\right)+J_{12}\left(\vec{R}_{q}, \vec{R}_{q^{\prime}}\right), \\
\Lambda_{9}=E_{1}=\sqrt{V^{2}+4 \Gamma^{2}}, & \Phi_{5}\left(\vec{R}_{q}, \vec{R}_{q^{\prime}}\right)=J_{11}\left(\vec{R}_{q}, \vec{R}_{q^{\prime}}\right)-J_{12}\left(\vec{R}_{q}, \vec{R}_{q^{\prime}}\right) ; \\
\Lambda_{12}=E_{2}=-\sqrt{V^{2}+4 \Gamma^{2}}, & \\
\Lambda_{15}=E_{3}=-V, & \\
\Lambda_{16}=E_{4}=V, &
\end{array}
$$

all other coefficients $\Lambda_{\lambda}$ and $\Phi_{\lambda}\left(\vec{R}_{q}, \vec{R}_{q^{\prime}}\right)$ in this case are equal to zero.

Taking a "non-interacting" part of the (1.3) Hamiltonian

$$
H_{0}=\sum_{\lambda \neq 1} \sum_{q} \Lambda_{\lambda} Y_{\lambda}\left(\vec{R}_{q}\right)
$$

as a reference system, for partition function functional in the collective variables representation one may obtain [6]:

$$
\begin{aligned}
Z=Z_{0}^{N} & \int\left(\mathrm{d} \rho_{\lambda}(\vec{k}, \nu)\right)^{N} \exp \left\{\sum _ { \lambda = 1 } ^ { 2 ^ { 2 f _ { 0 } } } \sum _ { k \leqslant B } \sum _ { \nu = 0 } ^ { \infty } \left[\frac{\beta}{2} \Phi_{\lambda}(\vec{k}) \rho_{\lambda}(\vec{k}, \nu) \rho_{\lambda}(-\vec{k},-\nu)\right.\right. \\
& \left.\left.-2 \beta \sqrt{N} h \rho_{\lambda}(\vec{k}, \nu) \delta_{\lambda 1} \delta(\vec{k}) \delta(\nu)\right]\right\} \\
& \times \int\left(\mathrm{d} \omega_{\lambda}(\vec{k}, \nu)\right)^{N} \exp \left\{\mathrm{i} 2 \pi \sum_{\lambda=1}^{2^{2 f_{0}}} \sum_{k, \nu} \omega_{\lambda}(\vec{k}, \nu) \rho_{\lambda}(\vec{k}, \nu)\right\} \\
& \times \exp \left\{\sum_{n=1}^{\infty} \frac{(-\mathrm{i} 2 \pi)^{n}}{n !} \sum_{\lambda_{1} \ldots \lambda_{n} \neq 1}^{2^{2 f_{0}}} \sum_{k_{1}, \ldots, k_{n} \leqslant B} \sum_{\nu_{1}, \ldots \nu_{n}=0}^{\infty} \mathcal{M}_{\lambda_{1} \ldots \lambda_{n}}\left(\vec{k}_{1}, \nu_{1}, \ldots \vec{k}_{n}, \nu_{n}\right)\right. \\
& \left.\times \omega_{\lambda_{1}}\left(\vec{k}_{1}, \nu_{1}\right) \ldots \omega_{\lambda_{n}}\left(\vec{k}_{n}, \nu_{n}\right)\right\} .
\end{aligned}
$$

Coefficients $\mathcal{M}_{\lambda_{1} \ldots \lambda_{n}}\left(\vec{k}_{1}, \nu_{1}, \ldots \vec{k}_{n}, \nu_{n}\right)$ are usual cumulant average values of the generalized transition operators $Y_{\lambda}\left(\vec{R}_{g}\right)$ products in the frequency-momentum representation

$$
\hat{\rho}_{\lambda}(\vec{k}, \nu)=\frac{1}{\beta} \int_{0}^{\beta} \mathrm{d} \beta^{\prime} \mathrm{e}^{-\mathrm{i} \beta^{\prime} \nu} \sum_{q=1}^{N} \mathrm{e}^{-\beta H_{0}} Y_{\lambda}\left(\vec{R}_{q}\right) \mathrm{e}^{\beta H_{0}} \mathrm{e}^{\mathrm{i} \vec{k} \vec{R}_{q}}
$$


In the (1.6), $\rho_{\lambda}(\vec{k}, \nu)$ are collective variables, corresponding to $\hat{\rho}_{\lambda}(\vec{k}, \nu) ; \nu=2 \pi / \beta n$ $(n=0, \pm 1, \pm 2, \ldots)$ are Matsubara's frequencies; $\beta=1 / k T, T$ is the absolute temperature; $k$ is the Boltzman constant; $B$ is the Brillouin zone boundary; $\Phi_{\lambda}(k)$ is a Fourier transform of $\Phi_{\lambda}\left(\vec{R}_{q}, \vec{R}_{q^{\prime}}\right) ; Z_{0}=\operatorname{Sp}\left\{\exp \left(-\beta H_{0}\right)\right\}$.

Since collective variables $\rho_{\lambda}(\vec{k}, \nu)$ decribe only a long-range part of the total Hamiltonian (short-range interactions are taken into account by $Z_{0}$ ) among different cumulants in (2.6), it is enough to take only the ones with $\lambda$, for which $\Phi_{\lambda}\left(\vec{R}_{g}, \vec{R}_{g^{\prime}}\right) \neq$ 0 . In the two-particle cluster system such indeceis are $\lambda=1$ and $\lambda=5$. Thus, all cluster cumulants in this case may be expressed by cumulant average values

$$
\left\langle R Y_{\lambda}\left(\vec{R}_{q_{1}}, \beta_{1}\right) Y_{\lambda}\left(\vec{R}_{q_{2}}, \beta_{2}\right) \ldots Y_{\lambda}\left(\vec{R}_{q_{m}}, \beta_{m}\right) \ldots\right\rangle_{0}^{\mathrm{c}}
$$

in which

$$
\begin{aligned}
Y_{1}\left(\vec{R}_{q}, \beta\right) & =\left(\begin{array}{cccc}
0 & 0 & b \mathrm{e}^{\beta E_{13}} & 0 \\
0 & 0 & a \mathrm{e}^{\beta E_{23}} & 0 \\
b \mathrm{e}^{-\beta E_{13}} & a \mathrm{e}^{-\beta E_{23}} & 0 & 0 \\
0 & 0 & 0 & 0
\end{array}\right), \\
Y_{5}\left(\vec{R}_{q}, \beta\right) & =\left(\begin{array}{cccc}
0 & 0 & 0 & -a \mathrm{e}^{\beta E_{14}} \\
0 & 0 & 0 & b \mathrm{e}^{\beta E_{24}} \\
0 & 0 & 0 & 0 \\
-a \mathrm{e}^{-\beta E_{14}} & b \mathrm{e}^{-\beta E_{24}} & 0 & 0
\end{array}\right),
\end{aligned}
$$

where

$$
\begin{aligned}
& a^{2}=\frac{1}{4}\left(1+\frac{V}{\sqrt{V^{2}+4 \Gamma^{2}}}\right), \quad b^{2}=\frac{1}{4}\left(1-\frac{V}{\sqrt{V^{2}+4 \Gamma^{2}}}\right), \\
& E_{i j}=E_{i}-E_{j} .
\end{aligned}
$$

$R$ in (2.9) is a symbol for "time" arrangement with respect to the inverse temperature $\beta$.

$$
\langle\ldots\rangle_{0}=\operatorname{Sp}\left\{\ldots \mathrm{e}^{-\beta H_{0}}\right\}\left[\operatorname{Sp}\left\{\mathrm{e}^{-\beta H_{0}}\right\}\right]^{-1} .
$$

It may be easily tested that the non-equal, identically to zero expressions (2.8), contain only an even number of operators $Y_{\lambda}\left(\vec{R}_{q}, \beta\right)$.

The calculation of $(2.8)$ is based on the method similar to the Vikh-BlokchDominisis theorem [7] with the commutation relation

$$
\begin{aligned}
{\left[Y_{\lambda}\left(\vec{R}_{q}\right), Y_{\lambda^{\prime}}\left(\vec{R}_{q^{\prime}}\right)\right] } & =\sum_{\mu} W_{\lambda \lambda^{\prime}}^{\mu} Y_{\mu}\left(\vec{R}_{q}\right) \delta\left(\vec{R}_{q}-\vec{R}_{q^{\prime}}\right) \\
W_{\lambda \lambda^{\prime}}^{\mu} & =\sum_{r, s, t}\left(U_{r s \lambda} U_{s t \lambda^{\prime}}-U_{s t \lambda} U_{r s \lambda^{\prime}}\right) U_{r t \mu}
\end{aligned}
$$

where $r, s, t$ are ordinary indicies and $\lambda, \lambda^{\prime}, \mu$ are double indices. 
For the $n$-th order cluster cumulant we have a formula:

$$
\begin{aligned}
\mathcal{M}_{2 n}^{(\lambda)} & \left(\vec{k}_{1}, \nu_{1}, \ldots, \vec{k}_{2 n}, \nu_{2 n}\right)=\left\langle R Y_{\lambda}\left(\vec{k}_{1}, \nu_{1}\right) \ldots Y_{\lambda}\left(\vec{k}_{2 n}, \nu_{2 n}\right)\right\rangle_{0}^{\mathrm{c}} \\
= & \frac{1}{\left(\beta^{2} N\right)^{n}} \sum_{q_{1}, \ldots, q_{2 n}} \mathrm{e}^{-\mathrm{i}\left(\vec{k}_{1} R_{q_{1}}+\ldots+\vec{k}_{2 n} \vec{R}_{q_{2 n}}\right)} \int_{0}^{\beta} \mathrm{d} \beta_{1} \ldots \int_{0}^{\beta} \mathrm{d} \beta_{2 n} \mathrm{e}^{-\mathrm{i}\left(\beta_{1} \nu_{1}+\ldots+\beta_{2 n} \nu_{2 n}\right)} \\
& \times\left\langle R Y_{\lambda}\left(\vec{R}_{q_{1}}, \beta_{1}\right) \ldots Y_{\lambda}\left(\vec{R}_{q_{2 n}}, \beta_{2 n}\right)\right\rangle_{0}^{\mathrm{c}} .
\end{aligned}
$$

The general form of the $(2 n)$-th order cumulant is:

$$
\begin{aligned}
& \mathcal{M}_{2 n}=\widetilde{\mathcal{M}}_{2 n}-\left[\frac{(2 n) !}{2 !(2 n-2) !} \widetilde{\mathcal{M}}_{2} \widetilde{\mathcal{M}}_{2 n-2}+\frac{(2 n) !}{4 !(2 n-4) !} \widetilde{\mathcal{M}}_{4} \widetilde{\mathcal{M}}_{2 n-4}\right. \\
& +\frac{(2 n) !}{6 !(2 n-6) !} \widetilde{\mathcal{M}}_{6} \widetilde{\mathcal{M}}_{2 n-6}+\ldots \\
& \left.+\frac{(2 n) !}{(2 p) !(2 n-2 p) !}\left(1-\frac{\delta_{n-2 p}}{2}\right)_{2 p \leqslant n} \widetilde{\mathcal{M}}_{2 p} \widetilde{\mathcal{M}}_{2 n-2 p}\right] \\
& +2 !\left[\frac{(2 n) !}{2 !(2 !)^{2}(2 m-4) !} \widetilde{\mathcal{M}}_{2}^{2} \widetilde{\mathcal{M}}_{2 n-4}+\frac{(2 n) !}{2 ! 4 !(2 n-6) !} \widetilde{\mathcal{M}}_{2} \widetilde{\mathcal{M}}_{4} \widetilde{\mathcal{M}}_{2 n-6}\right. \\
& +\frac{(2 n) !}{2 !(4 !)^{2}(2 n-8) !} \widetilde{\mathcal{M}}_{4}^{2} \widetilde{\mathcal{M}}_{2 n-8}+\frac{(2 n) !}{2 ! 6 !(2 n-8) !} \widetilde{\mathcal{M}}_{2} \widetilde{\mathcal{M}}_{6} \widetilde{\mathcal{M}}_{2 n-8} \\
& +\frac{(2 n) !}{4 ! 6 !(2 n-10) !} \widetilde{\mathcal{M}}_{4} \widetilde{\mathcal{M}}_{6} \widetilde{\mathcal{M}}_{2 n-10}+\frac{(2 n) !}{2 ! 8 !(2 n-10) !} \widetilde{\mathcal{M}}_{2} \widetilde{\mathcal{M}}_{8} \widetilde{\mathcal{M}}_{2 n-10} \\
& +\frac{(2 n) !}{2 !(6 !)^{2}(2 n-12) !} \widetilde{\mathcal{M}}_{6}^{2} \widetilde{\mathcal{M}}_{2 n-12}+\frac{(2 n) !}{4 ! 8 !(2 n-12) !} \widetilde{\mathcal{M}}_{4} \widetilde{\mathcal{M}}_{8} \widetilde{\mathcal{M}}_{2 n-12} \\
& \left.+\frac{(2 n) !}{2 ! 10 !(2 n-12) !} \widetilde{\mathcal{M}}_{2} \widetilde{\mathcal{M}}_{10} \widetilde{\mathcal{M}}_{2 n-12}+\ldots\right] \\
& -3 !\left[\frac{(2 n) !}{3 !(2 !)^{3}(2 n-6) !} \widetilde{\mathcal{M}}_{2}^{3} \widetilde{\mathcal{M}}_{2 n-6}+\frac{(2 n) !}{2 !(2 !)^{2} 4 !(2 n-8) !} \widetilde{\mathcal{M}}_{2}^{2} \widetilde{\mathcal{M}}_{4} \widetilde{\mathcal{M}}_{2 n-8}\right. \\
& +\frac{(2 n) !}{2 !(2 !)^{2} 6 !(2 n-10) !} \widetilde{\mathcal{M}}_{2}^{2} \widetilde{\mathcal{M}}_{6} \widetilde{\mathcal{M}}_{2 n-10} \\
& +\frac{(2 n) !}{2 ! 2 !(4 !)^{2}(2 n-10) !} \widetilde{\mathcal{M}}_{2} \widetilde{\mathcal{M}}_{4}^{2} \widetilde{\mathcal{M}}_{2 n-10} \\
& +\frac{(2 n) !}{2 !(2 !)^{2} 8 !(2 n-12) !} \widetilde{\mathcal{M}}_{2}^{2} \widetilde{\mathcal{M}}_{8} \widetilde{\mathcal{M}}_{2 n-12} \\
& +\frac{(2 n) !}{2 ! 4 ! 6 !(2 n-12) !} \widetilde{\mathcal{M}}_{2} \widetilde{\mathcal{M}}_{4} \widetilde{\mathcal{M}}_{6} \widetilde{\mathcal{M}}_{2 n-12} \\
& \left.+\frac{(2 n) !}{3 !(4 !)^{3}(2 n-12) !} \widetilde{\mathcal{M}}_{4}^{3} \widetilde{\mathcal{M}}_{2 n-12}+\ldots\right] \\
& +4 !\left[\frac{(2 n) !}{4 !(2 !)^{4}(2 n-8) !} \widetilde{\mathcal{M}}_{2}^{4} \widetilde{\mathcal{M}}_{2 n-8}\right. \\
& \left.+\frac{(2 n) !}{3 !(2 !)^{3} 4 !(2 n-10) !} \widetilde{\mathcal{M}}_{2}^{3} \widetilde{\mathcal{M}}_{4} \widetilde{\mathcal{M}}_{2 n-10}+\ldots\right]
\end{aligned}
$$




$$
+\ldots+(-1)^{n-1}(n-1) ! \frac{(2 n) !}{(2 !)^{n} n !} \widetilde{\mathcal{M}}_{2}^{n},
$$

where $\widetilde{\mathcal{M}}_{2 n}$ are redusible parts of (2.13). After a precise calculation of $(2.13),(2.14)$ we obtain a final formula for the $(2 n)$-th order cumulants

$$
\begin{aligned}
\mathcal{M}_{2 n}^{(\lambda)}\left(\vec{k}_{1}, \nu_{1}, \ldots \vec{k}_{2 n}, \nu_{2 n}\right)= & {\left[\frac{A^{(\lambda)}\left(E_{1}^{2}-E_{3}^{2}\right)}{2 \beta E_{1} Z_{0}}\right]^{n} C_{n-1}\left(x_{\lambda}\right) } \\
& \times \delta\left(\vec{k}_{1}+\ldots+\vec{k}_{2 n}\right) \delta\left(\nu_{1}+\ldots+\nu_{2 n}\right) .
\end{aligned}
$$

Here $C_{n-1}\left(x_{\lambda}\right)$ is a $(n-1)$-order polynomos

$$
\begin{aligned}
C_{n-1}\left(x_{\lambda}\right) & =\sum_{p=1}^{n}(-1)^{p-1} a_{p} x_{\lambda}^{n-p} \\
& =a_{1} x_{\lambda}^{n-1}-a_{2} x_{\lambda}^{n-2}+\ldots+(-1)^{p-1} a_{p} x_{\lambda}^{n-p}+\ldots+(-1)^{n-1} a_{n}, \\
x_{\lambda} & =\frac{B^{(\lambda)} Z_{0}}{\beta A^{(\lambda)}\left(E_{1}^{2}-E_{3}^{2}\right)}, \quad x_{\lambda}>0, \\
A^{(\lambda)} & =\frac{\mathrm{e}^{-\beta E_{2}}-\mathrm{e}^{\mp \beta E_{3}}}{\left(E_{2} \mp E_{3}\right)^{2}+\nu^{2}}+\frac{\mathrm{e}^{\mp \beta E_{3}}-\mathrm{e}^{-\beta E_{1}}}{\left(E_{1} \mp E_{3}\right)^{2}+\nu^{2}}, \\
B^{(\lambda)} & =\frac{\left(E_{1} \mp E_{3}\right)\left(\cosh \beta\left(E_{2} \mp E_{3}\right)-1\right)}{\left(E_{2} \mp E_{3}\right)^{2}+\nu^{2}}-\frac{\left(E_{2} \mp E_{3}\right)\left(\cosh \beta\left(E_{1} \mp E_{3}\right)-1\right)}{\left(E_{1} \mp E_{3}\right)^{2}+\nu^{2}}, \\
Z_{0} & =\sum_{\lambda} \mathrm{e}^{-\beta E_{\lambda}}=2 \cosh \beta V+2 \cosh \beta \sqrt{V^{2}+4 \Gamma^{2}} .
\end{aligned}
$$

$a_{p}$ coefficients are equal to the simple sum of corresponding coefficients of the $p$-th level separation in (2.14). So, for example

$$
\begin{aligned}
& m=1, \quad c_{0}=1, \\
& m=2, \quad c_{1}=x-3 \text {, } \\
& m=3, \quad c_{2}=x^{2}-15 x+30, \\
& m=4, \quad c_{3}=x^{3}-63 x^{2}+420 x-630, \\
& m=5, \quad c_{4}=x^{4}-255 x^{3}+4410 x^{2}-18900 x+22680, \\
& m=6, \quad c_{5}=x^{5}-1023 x^{4}+42240 x^{3}-395010 x^{2}+1247400 x-1247400, \\
& m=7, \quad c_{6}=x^{6}-4095 x^{5}+390390 x^{4}-7207200 x^{3}+45405360 x^{2} \\
& -113513400 x-97297200 \text {, } \\
& m=8, \quad c_{7}=x^{7}-16383 x^{6}+3554460 x^{5}-123513390 x^{4}+1394593200 x^{3} \\
& -6583777200 x^{2}+13621608000 x-10216206000 \text {. }
\end{aligned}
$$

Taking into account the factors connected with the normalization of $Y_{\lambda}\left(\vec{R}_{q}\right)$ operators $(\sqrt{2})^{2 m}$ and the cluster structure of the Hamiltonian 2 at $V=0 ; \Gamma=0$ case, for example

$$
\mathcal{M}_{\lambda \lambda \lambda \lambda}^{\text {Ising }}=\frac{1}{8}(\sqrt{2})^{2 \cdot 2} \cdot 2-\frac{3}{16}(\sqrt{2})^{2 \cdot 2} \cdot 2^{2}=-2
$$


one obtains all values of the classic Ising model cumulants:

$$
\begin{array}{llrl}
\mathcal{M}_{2} & =1, & \mathcal{M}_{10}=7936 \\
\mathcal{M}_{4}=-2, & \mathcal{M}_{12}=-353792, \\
\mathcal{M}_{6}=16, & \mathcal{M}_{14}=22368256 \\
\mathcal{M}_{8}=-272, & \mathcal{M}_{16}=-1903757312
\end{array}
$$

and so on. In the cluster case at $V=0, \Gamma=0$ we have:

$$
\begin{array}{llrl}
\mathcal{M}_{2} & =\frac{1}{4}=0.25, & \mathcal{M}_{10}=\frac{31}{64} \approx 0.4844, \\
\mathcal{M}_{4}=-\frac{1}{16}=-0.0625, & \mathcal{M}_{12}=-\frac{691}{256} \approx-2.6992, \\
\mathcal{M}_{6}=\frac{1}{16}=0.0625, & \mathcal{M}_{14}=\frac{87375}{4096} \approx 21.3318, \\
\mathcal{M}_{8}=-\frac{17}{128} \approx-0.1328, & \mathcal{M}_{16}=-\frac{798569}{4096} \approx-194.9631
\end{array}
$$

and so on. (2.19) demonstrate that at $V=0, \Gamma=0$ all cumulants possess a good sign and the (2.6) functional is signified correctely.

\section{Role of $V$ and $\Gamma$ parameters in the cluster cumulants be- haviour}

It may be easily seen that cumulants $\mathcal{M}_{2 n}\left(\vec{k}_{1}, \nu_{1}, \ldots \vec{k}_{2 n}, \nu_{2 n}\right)$ behaviour with respect to intracluster interaction $V$ and transverse field $\Gamma$ are absolutely different. For instance let's consider $\lambda=1$.

$$
\begin{aligned}
& V=0 \\
& \mathcal{M}_{2 n}=\frac{(\beta \Gamma)^{n} \sinh ^{n} 2 \beta \Gamma}{\beta^{2 n}\left(4 \Gamma^{2}+\nu^{2}\right)^{n}(\cosh 2 \beta \Gamma+1)^{n}} C_{n-1}(x), \\
& x=\frac{\sinh 2 \beta \Gamma}{\beta \Gamma} \geqslant 2 ; \\
& \Gamma=0 \\
& \mathcal{M}_{2 n}=\left(\frac{\mathrm{e}^{\beta V}}{4 \cosh \beta V}\right)^{m} C_{n-1}(y), \\
& y=\frac{2 \cosh \beta V}{\mathrm{e}^{\beta V}} \leqslant 2 .
\end{aligned}
$$

At large $\Gamma$ all cumulants starting from $n=2$ are divergent. But at large $V$ they are finite. So, these two types of interactions play different role in the cluster system stability formation.

The cumulants increasing at $\Gamma \rightarrow \infty$ is not a unique danger for (2.6) functional. Because $C_{n-1}(x)$ is the $(n-1)$-order polynomos, the higher order cumulants $(n \geqslant$ 
Table 1. The first real root of polynomos $\mathrm{C}_{n-1}(x)$ and the limited values of transverse field intensity (for short range interaction absence case) which satisfy the investigated system stability.

\begin{tabular}{|c|c|c|c|c|c|c|c|c|}
\hline$m$ & 1 & 2 & 3 & 4 & 5 & 6 & 7 & 8 \\
\hline$x$ & - & 3.0 & 2.3765 & 2.2017 & 2.1264 & 2.0868 & 2.0634 & 2.0482 \\
\hline$\beta \Gamma_{l}$ & $\infty$ & 0.8111 & 0.5175 & 0.3833 & 0.3049 & 0.2534 & 0.2171 & 0.1895 \\
\hline
\end{tabular}

2 ) become a non-monotonous function of the $x$-parameter $(2.16)$, so $C_{n-1}(x)$ may change its sign at certain values of $V$ and $\Gamma$. As a result the functional (2.6) becomes infinite at certain values of these parameters and temperature. Evidently, the first root of $C_{n-1}(x)$ (for fixed $T$ ) determine a region of physically possible values of those parameters.

Introducing $A^{(\lambda)}$ and $B^{(\lambda)}$ into $x$ (2.16) one may test that $x$ depends on Matsubara frequency very slightly. So, for the analytic regard of some first polynomos $C_{n-1}(x)$ it is possible to put $\nu=0$. In this approximation:

$$
\begin{aligned}
x= & \frac{\cosh \beta V+\cosh \beta \sqrt{V^{2}+4 \Gamma^{2}}}{\beta \Gamma^{2}} \\
\times & {\left[\sqrt{V^{2}+4 \Gamma^{2}}\left(V^{2}+\Gamma^{2}\right)\left(\cosh \beta V \cosh \beta \sqrt{V^{2}+4 \Gamma^{2}}-1\right)\right.} \\
& \left.-V\left(V^{2}+3 \Gamma^{2}\right) \sinh \beta V \sinh \beta \sqrt{V^{2}+4 \Gamma^{2}}\right] \\
\times & {\left[\left(V^{2}+2 \Gamma^{2}\right) \sinh \beta \sqrt{V^{2}+4 \Gamma^{2}}+V \sqrt{V^{2}+4 \Gamma^{2}}\left(\cosh \beta \sqrt{V^{2}+4 \Gamma^{2}}-\mathrm{e}^{\beta V}\right)\right]^{-1} . }
\end{aligned}
$$

The first real root $(x>0)$ of polynomos (2.16) for $m \leqslant 8$ and the corresponding values of $\beta \Gamma_{l}$ (at $V=0$ ), calculated from the second equation in (3.1), are presented in table 1 . table 1

The numerical calculations for $m>8$ are rather cumbersome, but it may be prooved that

$$
\lim _{m \rightarrow \infty} C_{m-1}(x)=0, \quad \text { at } x=2 .
$$

The limiting value $\beta \Gamma_{l}$ corresponds to the situation when cluster cumulants change their sign and the partition function functional becomes infinite, so the system investigated becomes unstable. The limiting value $\Gamma_{l}$ nonlineary grows when temperature falls down. At large $m \beta \Gamma_{l} \rightarrow 0$. It means when $V=0$ the total functional (1.6) is convergable only for $\Gamma=0$. Transverse field, as is well-known, destroys the system at low temperatures.

Absolutely different situation takes place when $\Gamma=0$. In this case polynomos $C_{m-1}(x)$ have no roots in the range $0<V<\infty$, so all cluster cumulants possess a good sign and the cluster system is stable at every temperature.

The situation when both $V \neq 0$ and $\Gamma \neq 0$ is, naturally, more complicated. The dependence of $\Gamma_{l}$ on $V$ at different temperatures is presented in figure 1 and 


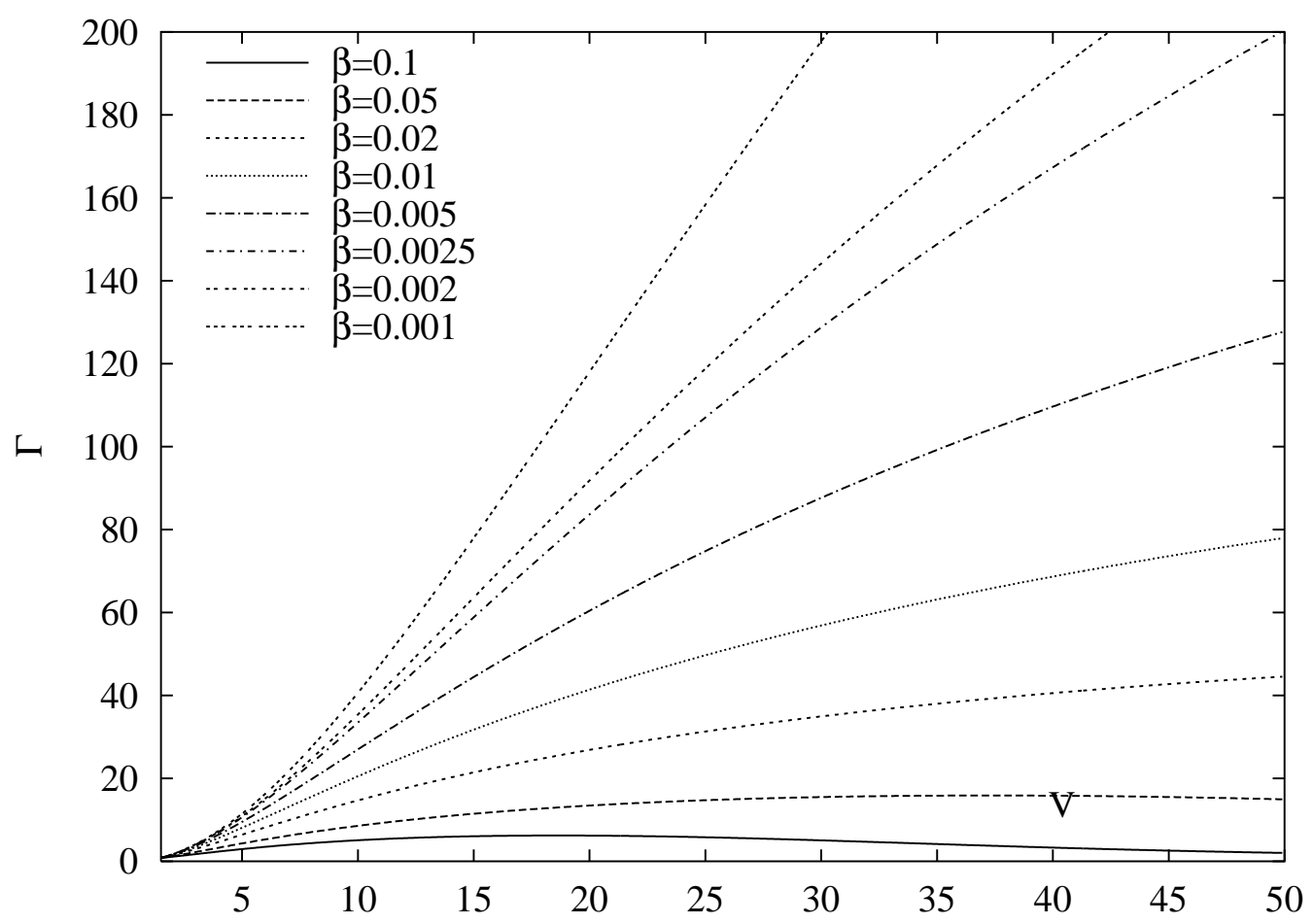

Figure 1. The limit value of the transverse field $\Gamma_{l}$ dependence on the intracluster interaction $V$ at different values of inverse temperature $\beta$.

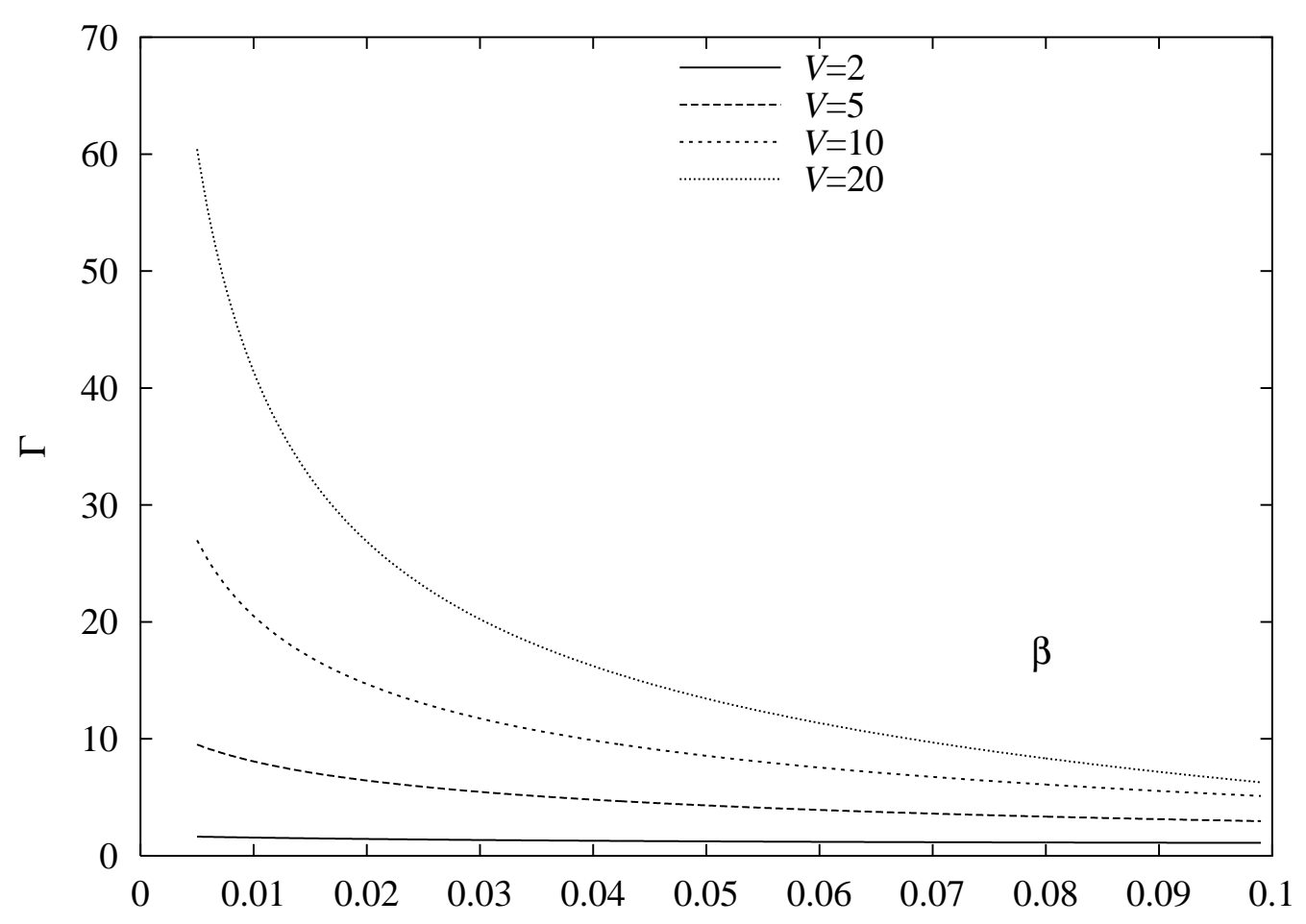

Figure 2. The limit value of the transverse field $\Gamma_{l}$ dependence on the inverse temperature $\beta$ at different values of the intracluster interaction $V$. 
the dependence of $\Gamma_{l}$ on $\beta$ at different $V$ is presented in figure 2. One can see that there is a limited value $\Gamma_{l}$ which divides $\Gamma, V$ (or $\Gamma, T$ ) diagram of state into two regions at every value of temperature and intracluster interactions. In the first one $\left(\Gamma<\Gamma_{l}\right)$ the functional (2.6) is defined correctly and the system remains in the stable state. Upper of the line $\Gamma=\Gamma_{l}$ cluster cumulants change their sign, functional (2.6) becomes divergent and the system investigated loses its stability. Correspondingly, calculated on the base of (2.6) functional thermodynamic functions will demonstrate unphysical behaviour. It must be noted that the situation discussed here takes place only for non-Gaussian approximation in (2.6) (when $m \geqslant 2$ ).

In practice, the calculation on the base of (2.6) functional envisages the usage of a limited (i.e. ussually not high) power of $\omega_{\lambda}(\vec{k}, \nu)$ variables. It is reasonable that even the first non-Gaussian approximation leads to a certain nonphysical behaviour of thermodynamic function of de Gennes model at low temperatures. Temperature points of unphysical behaviour (anomaly of polarization, dielectric susceptibility, heat capacity etc.) are called anti-curie points [3]. It must be noted that minimization procedure, used in [3] in the framework of self-consistent cluster approximation in its origin is close to taking into account some higher order correlation effects like non-Gaussian fourth order distribution in (2.6).

\section{References}

1. Yukhnovskii I.R. Phase Transition of the Second Order. Collective Variables Method. Singapore, World. Scientific, 1987.

2. Korynevskii N.A. // TMPh, 1983, vol. 55, p. 291 (in Russian).

3. Vaks V.G. Introduction into Microscopic Theory of Ferroelectricity. Moskow, Nauka, 1973 (in Russian).

4. Stasyuk I.V., Levitskii R.R., Korynevskii N.A. // Phys. Stat. Sol. (b), 1979, vol. 91, p. 541.

5. Yukhnovskii I.R., Korynevskii N.A. // Phys. Stat. Sol. (b), 1989, vol. 153, p. 583.

6. Korynevskii N.A. On the Maximum Transverse Field Magnitude in the Quantum Cluster Model. Preprint of the Institute for Condensed Matter Physics, ICMP-9909U, Lviv, 1999, 20 p. (in Ukrainian).

7. Abrikosov A.A., Gor'kov L.P., Dzialoshynskii I.E. Methods of Quantum Field Theory in Statistic Physics. Moskow, Izd. Phys.-Math., 1962 (in Russian). 


\title{
Про особливості кумулянтного розкладу для Функціоналу статистичної суми кластерної моделі де Жена
}

\author{
М.А.Кориневський \\ Інститут фізики конденсованих систем НАН України, \\ 79011 Львів, вул. Свєнціцького, 1 \\ Отримано 16 лютого 2000 p.
}

Досліджується проблема функціонального зображення функціонала статистичної суми кластерної моделі де Жена в методі колективних змінних. На противагу до звичайної моделі Ізінга коефіцієнти отриманого функціонала статистичної суми (кластерні кумулянти) залежать від температури $T$ і поперечного поля $Г$. Внаслідок цього при низьких тмпературах виникає строге обмеження на величину параметра Г . Отримано рівняння для максимального значення $\Gamma_{l}$, температури і величини короткосяжних внутрікластерних взаємодій $V$. Знайдено розв'язки цього рівняння.

Ключові слова: кластерна модель де Жена, функціонал статистичної суми, фазові перетворення.

PACS: 05.60.+W, 05.70.Ln, 05.20.Dd, 52.25.Dg, 52.25.Fi 
